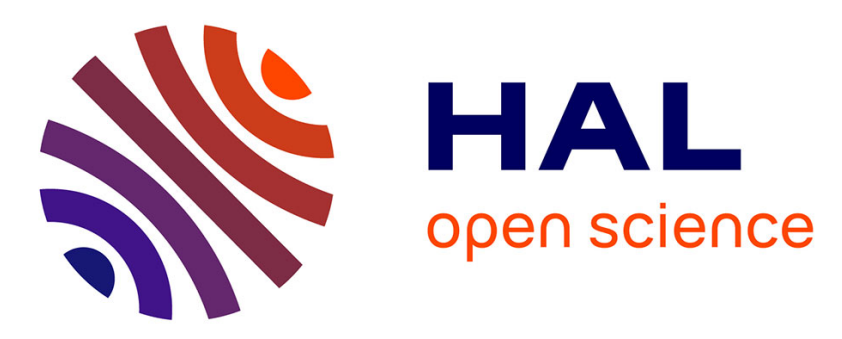

\title{
3D CFD in complex vascular systems: A case study
}

Olivia Miraucourt, Olivier Génevaux, Marcela Szopos, Marc Thiriet, Hugues

Talbot, Stéphanie Salmon, Nicolas Passat

\section{To cite this version:}

Olivia Miraucourt, Olivier Génevaux, Marcela Szopos, Marc Thiriet, Hugues Talbot, et al.. 3D CFD in complex vascular systems: A case study. International Symposium on Biomedical Simulation (ISBMS), 2014, Strasbourg, France. pp.86-94, 10.1007/978-3-319-12057-7_10 . hal-01695067

\section{HAL Id: hal-01695067 \\ https://hal.univ-reims.fr/hal-01695067}

Submitted on 15 Feb 2018

HAL is a multi-disciplinary open access archive for the deposit and dissemination of scientific research documents, whether they are published or not. The documents may come from teaching and research institutions in France or abroad, or from public or private research centers.
L'archive ouverte pluridisciplinaire HAL, est destinée au dépôt et à la diffusion de documents scientifiques de niveau recherche, publiés ou non, émanant des établissements d'enseignement et de recherche français ou étrangers, des laboratoires publics ou privés. 


\title{
3D CFD in Complex Vascular Systems: A Case Study ${ }^{\star}$
}

\author{
Olivia Miraucourt ${ }^{1,2}$, Olivier Génevaux ${ }^{3}$, Marcela Szopos ${ }^{4}$, Marc Thiriet ${ }^{5}$, \\ Hugues Talbot ${ }^{2}$, Stéphanie Salmon ${ }^{1}$, and Nicolas Passat ${ }^{6}$ \\ ${ }^{1}$ Université de Reims Champagne-Ardenne, LMR, France \\ ${ }^{2}$ Université Paris-Est, ESIEE, CNRS, LIGM, France \\ ${ }^{3}$ Université de Strasbourg, CNRS, ICube, France \\ ${ }^{4}$ Université de Strasbourg, CNRS, IRMA, France \\ ${ }^{5}$ Université Paris 6, CNRS, LJLL, France \\ ${ }^{6}$ Université de Reims Champagne-Ardenne, CReSTIC, France
}

\begin{abstract}
Modeling the flowing blood in vascular structures is crucial to perform in silico simulations in various clinical contexts. This remains however an emerging and challenging research field, that raises several open issues. In particular, a compromise is generally made between the completeness of the simulation and the complicated architecture of the vasculature: reduced order simulations (lumped parameter models) represent vascular networks, whereas detailled models are devoted to small regions of interest. However, technical improvements enable targeting of compartments of the blood circulation rather than focusing on vascular branched segments. This article aims at investigating the cerebral flow in the entire venous drainage that can be reconstructed from medical imaging.
\end{abstract}

\section{Introduction}

The cerebral vasculature is a highly complex three-dimensional network, composed by three successive compartments: arteries, capillaries and veins. The arterial cerebral vasculature is characterized by a relatively invariant anatomic pattern $[1,2]$. The major part of the current literature on macro/mesoscopic modeling and simulation of blood flow in the brain has focused on this first compartment [3]. By contrast, venous cerebral system was rarely studied and still remains not well understood. In particular, several difficulties are to be considered: the lack of parallelism between arterial and venous circulations; the asymmetric and considerably more various pattern of the venous network compared to the arterial; the highly individual variations of the venous outflow.

In this challenging context, flowing blood simulation is highly relevant for various clinical purposes. It can allow diagnosis and follow-up of aneurysms [4], or predict the effects of stenting [5] or coiling [6] procedures. In order to be actually useful, blood flow modeling has to fit at best the physical rules of fluid dynamics, with respect to

\footnotetext{
* This research was funded by a grant from the Région Champagne-Ardenne and by Agence Nationale de la Recherche (Grant Agreement ANR-12-MONU-0010). MRI images were provided by the In Vivo Imaging Platform of Université de Strasbourg. Computing resources were provided by the HPC Center of Université de Reims Champagne-Ardenne.
} 
physiological assumptions. As a consequence, by opposition to many clinical applications where virtual reality provides satisfactory paradigms, it is mandatory to consider mathematical approaches relying on numerical analysis [7]. This strategy presents a higher computational cost, but also a higher - and crucial - physical reliability.

Computational fluid dynamics has evolved with the development of new numerical schemes and high performance computing. However, despite many breakthroughs, open problems still exist. They derive from computational cost issues, but also from the difficulty to accurately model the multiple physiological assumptions. In this methodological framework, two dual strategies are generally considered.

The first strategy consists of performing reduced order simulations, i.e., to consider $1 \mathrm{D}$ models of the 3D vessels. The strong decrease of space complexity allows the numerical solvers to deal with complex vascular networks, and to provide accurate - yet macroscopic - behavior of the global flowing blood. This paradigm has been considered to handle the vascular networks of specific organs [8], but also of the whole human body [9-11]. However, local values of vascular resistances and compliances remain, in general, unknown in the context of personalized studies.

The second strategy consists of performing complete computational fluid dynamics simulations, i.e., to consider the 3D geometry of the vessels, and to simulate fine blood flow patterns. In particular, it has been demonstrated [12] that a satisfactory agreement is obtained by 1D models during diastole, but significant differences appear during systole, partially explained by the inability of the currently used 1D models to take into account secondary flow features, vessel curvature, etc. In 3D models, the huge size of the induced equation systems generally leads to focus on vessel samples. Classical use cases are then related to large vessels [13] or to vascular pathologies $[4,14]$ where the considered vascular geometries are vessel segments. Unfortunately, sampling the vessels induces a bias that alters the correctness of the flow information [15].

This motivates the development of simulation strategies that accurately handle both complicated vascular geometries and 3D blood flow models. Approaches based on mixed 3D/1D computational fluid dynamics models [16] are a first - yet partial - step towards this goal. (The use of 3D/1D coupled models allows to study how local and global hemodynamics phenomena are linked, for instance, the carotids can be interfaced with the more complex arterial network $[17,18]$.) Regarding a fully 3D context, few attempts have been proposed for the cerebral arterial [19] and venous [20] networks. Both were preliminary, and the latter essentially dealt with the initialization of the simulation, i.e. a stationary Stokes problem.

Our efforts toward this goal have been carried out by following three fundamental guidelines: (i) developing/using open-source softwares, in order to have a full handling of the underlying methods, and to provide guarantees in terms of current and future availability as well as reproducible research; (ii) proving the numerical correctness of the developed solvers, giving quality guarantees both in terms of reliability, reproducibility and incrementality, in contrast to many previous approaches based on commercial software packages; and (iii) considering a cautious physically-oriented modeling of the flowing blood behavior, to obtain not only realistic, but also reliable - and thus hopefully useful - results. 


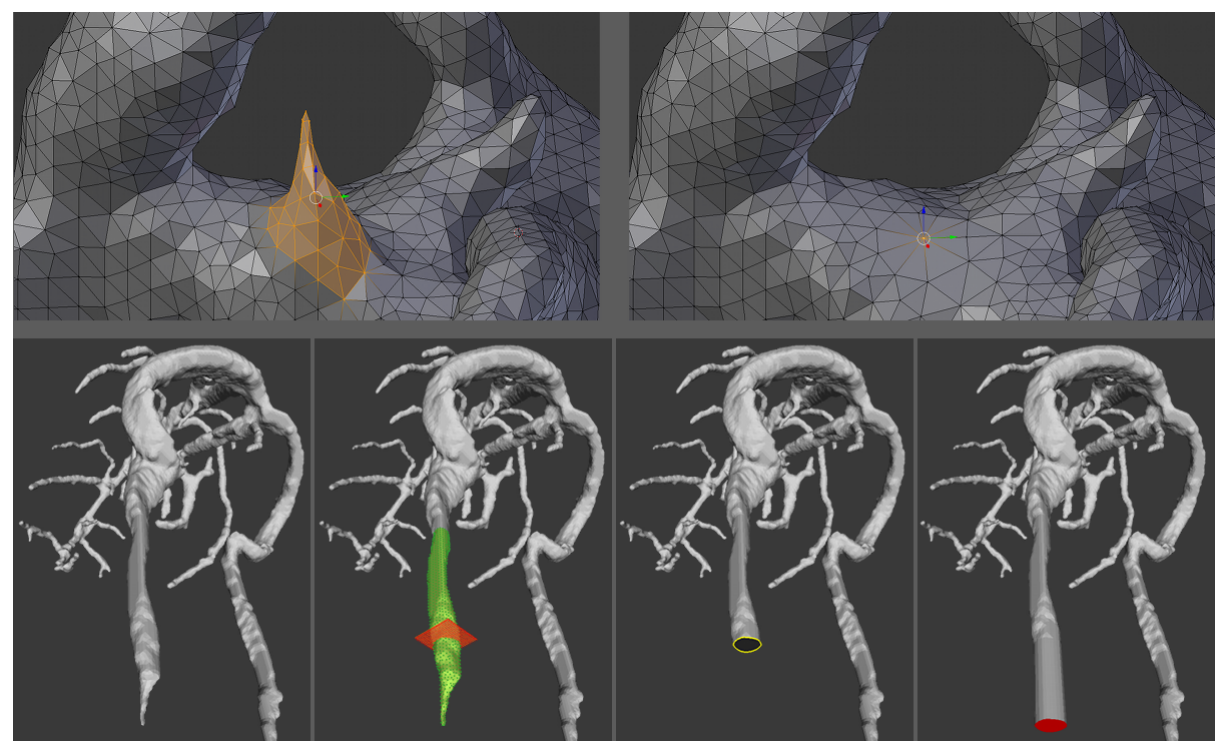

Fig. 1. First row: geometry correction of a $2 \mathrm{D}$ mesh. Second row: processing of the inputs and outputs (identification, cutting and extrusion).

The article is structured as follows. Sec. 2 summaries the chain to obtain a realistic geometry. Sec. 3 is devoted to the modeling of the venous network in order to obtain the adequate equations to solve. In Sec. 4, we give details on the considered numerical approaches and algorithms. We present in Sec. 5 numerical results on analytical solutions and on a realistic case. We gather in Sec. 6 some concluding remarks.

\section{Geometric Modeling}

The first step of our scheme deals with image processing and mesh generation issues. It takes as input a 3D MRA (Magnetic Resonance Angiography) data, whose resolution is sufficient at a macroscopic scale. Images were acquired on a whole-body scanner (Siemens Magnetom Verio 3.0 T, gradient slope $=200 T / \mathrm{m} / \mathrm{s}$, flow encoding sequence, $\mathrm{TR}=42.7 \mathrm{~ms}, \mathrm{TE}=6.57 \mathrm{~ms}$, resolution $=0.4 \times 0.4 \times 0.8 \mathrm{~mm}^{3}$ ).

An example-based segmentation method [21] is used to extract the venous 3D digital volume. Marching-cube is then used to compute their boundaries as 2D meshed surfaces. These meshes cannot be used straight-away to generate the 3D computational fluid dynamics meshes. Indeed, (i) local geometric corrections are needed before being suitable for simulation, and (ii) it is required to identify domain input/outputs. The first task is crucial, as geometric artifacts can remain locally despite the efforts put into the segmentation, such as spikes off the main surface due to small branching vessels, undesired small branches, small disconnected components, etc. The second task is required so one can latter setup appropriate boundary conditions for the numerical model on each 
venous tree tip (Fig. 1). Due to the necessity to interactively inspect the mesh, we tackled these tasks with a workflow supported by a combination of off-the-shelf tools such as the BLENDER modeling software and appropriately developed software. The former is geared towards general mesh editing to fix areas that are deemed to be problematic while the latter is more dedicated to perform problem-specific tasks efficiently, for instance inputs and outputs labeling and reshaping. Finally, we smooth the $2 \mathrm{D}$ mesh with MMGs before building the 3D tetrahedral simulation mesh with MMG3D. The size of mesh, with respect to the number of degrees of freedom, is chosen at this step.

\section{Physical Modeling}

Our aim is now to derive an appropriate model for the large cerebral veins located in the leptomeninges down to the extracranial drainage. At the macroscopic scale, the brain venous network (see illustration) is composed by - input - veins (vein of Galen (7); internal cerebral vein (8); basilar vein (9); superior cerebral veins (10); superior anastomotic veins (11)) draining the blood into the superior sagittal sinus (2) and the straight sinus (3), until their confluence (4). The blood then passes into the transverse (5) and sigmoid parts (6) of the lateral sinuses, and reaches an extracranial area, composed of the - output internal jugular veins (1) [1].

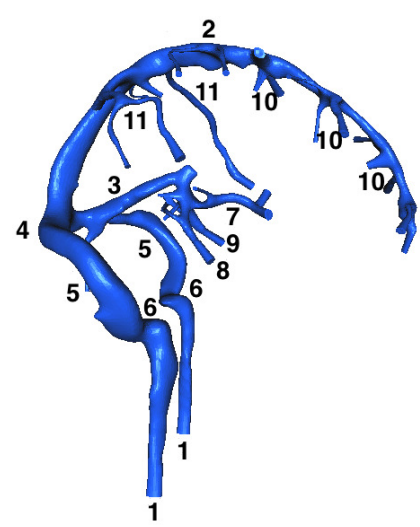

Concerning the flow in large/medium-sized cerebral veins, we adopt standard assumptions: (i) the blood density is constant; (ii) the flow is incompressible and isothermal. Blood is also supposed to be Newtonian [3,22] as, in the absence of blood stagnation regions, rouleaux of red blood capsules are formed neither in the upstream microcirculation (particle train flow) nor in veins. Moreover, in such veins, flowing cells do not deform. Hence thixotropic, viscoelastic, and shear thinning features are omitted in the context of a drainage circuit that receives a steady, laminar flow at its entrance.

Another important issue is the relevance of using either a complex fluid-structure interaction model or a fluid model. Intracranial veins that run through the leptomeninges (mostly water) are quite constrained between a nearly incompressible brain and the rigid skull. They are thus not susceptible to experience large deformations, and are considered as rigid. (More generally neglecting wall motion has been shown to be an acceptable hypothesis when studying intracranial arterial blood flow [23].)

The boundary conditions are: (i) inflow: a steady profile (constant velocity of small magnitude, due to microcirculation exit, far from the thorax hence far from the effects of breathing and cardiac pumping [24]); (ii) outflow: homogeneous natural conditions; (iii) lateral boundary: no-slip condition, since walls are assumed to be rigid.

Computed orders of the Reynolds $(90 \rightarrow 232)$, Stokes $(1.10 \rightarrow 3.84)$ and Strouhal numbers $(0.013 \rightarrow 0.063)$ show the potential importance of the convective forces and flow unsteadiness. In conclusion, the mathematical model that we consider is the 
Navier-Stokes equations with mixed boundary conditions

$$
\left\{\begin{aligned}
\partial_{t} \mathbf{u}+\mathbf{u} \cdot \boldsymbol{\nabla u}-v \Delta \mathbf{u}+\nabla p & =\mathbf{f} & & \text { in } \Omega \times(0, T) \\
\boldsymbol{\nabla} \cdot \mathbf{u} & =0 & & \text { in } \Omega \times(0, T) \\
\mathbf{u} & =\mathbf{g}_{1} & & \text { on } \Gamma_{D} \times(0, T) \\
v \frac{\partial \mathbf{u}}{\partial \mathbf{n}}-p \mathbf{n} & =\mathbf{g}_{2} & & \text { on } \Gamma_{N} \times(0, T) \\
\left.\mathbf{u}\right|_{t=0} & =\mathbf{u}_{0} & & \text { in } \Omega
\end{aligned}\right.
$$

where $\Omega \subset \mathbb{R}^{3}$ is a bounded domain with a Lipschitz-continuous boundary $\Gamma,(0, T)$ is a finite time interval. We recall that $\mathbf{u}$ is the fluid velocity, $p$ its pressure and $v$ its kinematic viscosity. We suppose that $\Gamma$ consists of two measurable parts: $\Gamma_{D}$, where Dirichlet boundary conditions are imposed, and $\Gamma_{N}$, where Neumann boundary conditions are prescribed. We denote by $\mathbf{n}$ the unit outward normal vector to $\Omega$ on its boundary $\Gamma$. The Dirichlet boundary conditions correspond in the non-homogeneous case to a velocity $\mathbf{g}_{1}$, generally prescribed at the inlets, and in the homogeneous case to the non-slip boundary condition prescribed at the vessel wall; the function $\mathbf{g}_{2}$ corresponds to the normal component of the stress tensor, prescribed at the outlets.

\section{Numerical methods}

To solve Eq. (1), we consider two numerical schemes presenting dual properties. The first $[25,3]$ considers a discretization involving a time-scheme based on the characteristics method and a spatial discretization of finite element type. Theoretically expected convergence in space for velocity and pressure error are of order 3 and 2 (resp. 2 and 1) for the $L^{2}$-norm (resp. the semi-norm $H^{1}$ ). The characteristics method is proven to be unconditionally stable and expected to be of order 1 in time.

The second method [26] belongs to the class of truly consistent splitting schemes [27], which decouples the computation of the velocity and pressure. It computes an approximate solution to the Navier-Stokes equations by solving at each time step a Poisson equation for the pressure and a heat equation for each component of the velocity. Its temporal accuracy was numerically assessed in 2D analytical cases [26] and observed to be of order 2 for both velocity and pressure. The spatial convergence was not evaluated, but second order accuracy for the space error is also expected.

Our implementations rely on the FrEEFEM++ library [28], that enables to run algorithms on parallel architectures. All codes and details are freely available on-line ${ }^{1}$.

We performed a validation on a gold-standard CFD problem with known analytical solutions [29]. The results (Fig. 2, log scale) confirm the numerical soundness of the schemes and our implementation. Our analysis shows that the second enjoys high-order convergence properties. However, the first remains faster in its current implementation.

\section{Results}

For our experiments on a real vascular model, a finite element mesh of 237438 tetrahedra was generated. Since blood comes from microcirculation (quasi-steady/steady

\footnotetext{
${ }^{1}$ http://numtourcfd.univ-reims.fr
} 

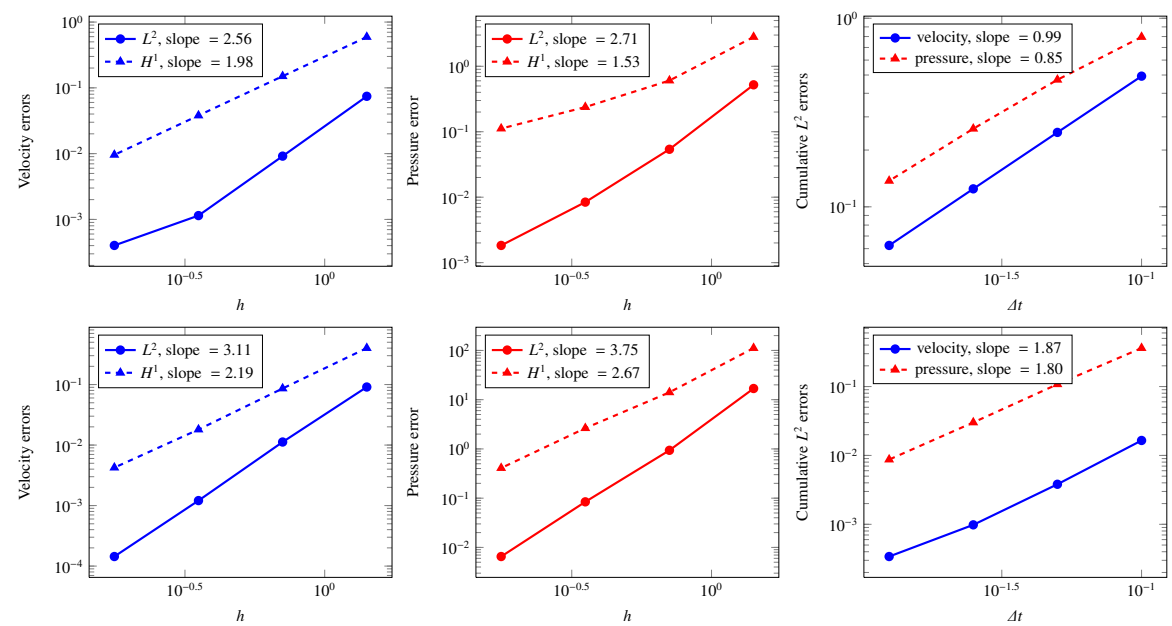

Fig. 2. Convergence curves of numerical errors. First row: [3]; second row: [26]. From left to right: space errors for velocity; space error for pressure; time errors for velocity and pressure.

Stokes flow), we use a steady profile of small magnitude $(28 \mathrm{~mm} / \mathrm{s})$, at the inflow. The no-slip condition was imposed on the wall boundaries, that are assumed to be rigid. For the outflow, we used a free traction boundary condition at the jugular vein extremities.

We performed 1000 iterations, with a time-step equal to $10^{-3} s$, in 14 hours on 16 processors, thus providing a simulation of 1 second of non-steady blood flow within cerebral venous network. Results obtained with this method are illustrated in Fig. 3-4.

Flows calculated at inlets and at outlets are identical (mass conservation property), with a value of $6227 \mathrm{~mm}^{3} / \mathrm{s}$, corresponding to physiological data [2]. Complex pattern flows are present and an asymmetric behavior in the two outlet branches can be observed, due to the physiological asymmetric pattern of the venous network. The depicted pressure should be interpreted relative to a reference pressure, applied to the models outlets; the obtained pressure drop of approximately $3.5 \mathrm{~mm} \mathrm{Hg}$ is in a physiological range [11]. It is worth noting that, since we use a rigid-walls model, the results are identical regardless of which value is used as a reference.

\section{Conclusion}

Many questions are raised by this work. Among the most crucial, one can cite the potential influence of the beating heart and the respiration on the outflow. These physiological phenomena should be taken into account in the outflow boundary conditions. In addition, the boundary conditions should take into account the position - horizontal or vertical - of the subject [30]. We also have to work on the collapsibility of the jugular veins. All these considerations may further lead to a better modeling.

Beyond the numerical validations with respect to analytical results, a main issue remains the validations in the real vascular network. If physical phantoms can be con- 


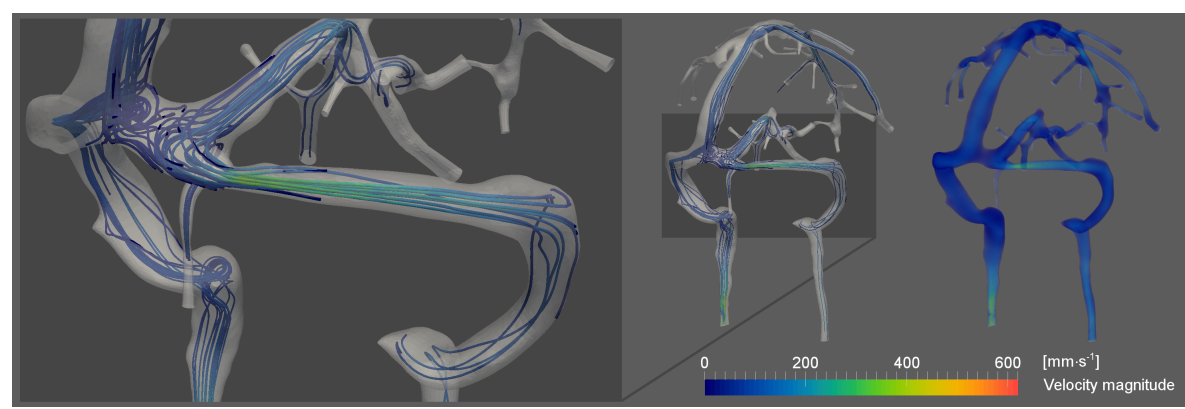

Fig. 3. Navier-Stokes simulation (iteration 250/1 000): streamlines and magnitude of velocity.

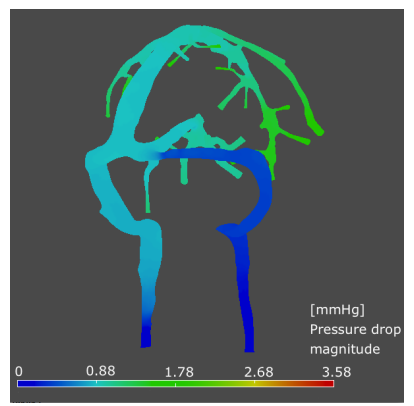

Fig. 4. Navier-Stokes simulation (iteration 250/1 000): magnitude of pressure drop field.

sidered to reach that goal in the case of simple vascular structures, such approaches are hardly tractable for complex networks. The development of virtual angiography, that simulates the physical image acquisition sequences, in order to create realistic synthetic data, provides a promising perspective. The case of Computed Tomography Angiography has already been pioneered [31]. The case of MRA is an emerging research field that constitutes one of our very goals, and motivates our use of MRA images.

\section{References}

1. Schaller, B.: Physiology of cerebral venous blood flow: From experimental data in animals to normal function in human. Brain Res Brain Res Rev 46 (2004) 243-260

2. Stoquart-Elsankari, S., Lehmann, P., Villette, A., Czosnyka, M., Meyer, M.E., Deramond, H., Balédent, O.: A phase-contrast MRI study of physiologic cerebral venous flow. J Cereb Blood F Met 29 (2009) 1208-1215

3. Formaggia, L., Quarteroni, A., Veneziani, A., eds.: Cardiovascular Mathematics. Volume 1 of MS \& A. Springer (2009)

4. Cebral, J.R., Castro, M.A., Appanaboyina, S., Putman, C.M., Millan, D., Frangi, A.F.: Efficient pipeline for image-based patient-specific analysis of cerebral aneurysm hemodynamics: Technique and sensitivity. IEEE T Med Imaging 24 (2005) 457-467 
5. Larrabide, I., Kim, M., Augsburger, L., Cruz Villa-Uriol, M., Rüfenacht, D., Frangi, A.F.: Fast virtual deployment of self-expandable stents: Method and in vitro evaluation for intracranial aneurysmal stenting. Med Image Anal 16 (2012) 721-730

6. Morales, H.G., Larrabide, I., Geers, A.J., San Román, L., Blasco, J., Macho, J.M., Frangi, A.F.: A virtual coiling technique for image-based aneurysm models by dynamic path planning. IEEE T Med Imaging 32 (2013) 119-129

7. Taylor, C.A., Figueroa, C.A.: Patient-specific modeling of cardiovascular mechanics. Annu Rev Biomed Eng 11 (2009) 109-134

8. Ho, H., Mithraratne, K., Hunter, P.: Numerical simulation of blood flow in an anatomicallyaccurate cerebral venous tree. IEEE T Med Imaging 32 (2013) 85-91

9. Reymond, P., Merenda, F., Perren, F., Rüfenacht, D., Stergiopulos, N.: Validation of a onedimensional model of the systemic arterial tree. Am J Physiol 297 (2009) 208-222

10. Blanco, P.J., Leiva, J.S., Buscaglia, G.C.: A black-box decomposition approach for coupling heterogeneous components in hemodynamics simulations. Int J Num Meth Biomed Eng 29 (2013) 408-427

11. Müller, L.O., Toro, E.F.: A global multiscale mathematical model for the human circulation with emphasis on the venous system. Int J Num Meth Biomed Eng (In Press)

12. Xiao, N., Alastruey, J., Figueroa, C.A.: A systematic comparison between 1-D and 3-D hemodynamics in compliant arterial models. Int J Num Meth Biomed Eng 30 (2014) 204231

13. Camara, O., Mansi, T., Pop, M., Rhode, K.S., Sermesant, M., Young, A.A., eds.: STACOM. Volume 8330 of LNCS., Springer (2014)

14. Boissonnat, J.D., Chaine, R., Frey, P., Malandain, G., Salmon, S., Saltel, E., Thiriet, M.: From arteriographies to computational flow in saccular aneurisms: The INRIA experience. Med Image Anal 9 (2005) 133-143

15. Sato, K., Imai, Y., Ishikawa, T., Matsuki, N., Yamaguchi, T.: The importance of parent artery geometry in intra-aneurysmal hemodynamics. Med Eng Phys 30 (2008) 774-782

16. Ho, H., Sorrell, K., Peng, L., Yang, Z., Holden, A., Hunter, P.: Hemodynamic analysis for transjugular intrahepatic portosystemic shunt (TIPS) in the liver based on a CT-image. IEEE T Med Imaging 32 (2013) 92-98

17. Passerini, T., de Luca, M., Formaggia, L., Quarteroni, A., Veneziani, A.: A 3D/1D geometrical multiscale model of cerebral vasculature. J Eng Math 64 (2009) 319-330

18. Blanco, P.J., Pivello, M.R., Urquiza, S.A., Feijoo, R.A.: On the potentialities of 3D-1D coupled models in hemodynamics simulations. J Biomech 42 (2009) 919-930

19. Mut, F., Wright, S., Ascoli, G., Cebral, J.R.: Characterization of the morphometry and hemodynamics of cerebral arterial trees in humans: A preliminary study. In: CMBE. (2011) 87-90

20. Miraucourt, M., Salmon, S., Szopos, M., Thiriet, M.: Blood flow simulations in the cerebral venous network. In: CMBE. (2013) 187-190

21. Dufour, A., Tankyevych, O., Naegel, B., Talbot, H., Ronse, C., Baruthio, J., Dokládal, P., Passat, N.: Filtering and segmentation of 3D angiographic data: Advances based on mathematical morphology. Med Image Anal 17 (2013) 147-164

22. Thiriet, M.: Cell and Tissue Organization in the Circulatory and Ventilatory Systems. Springer (2011)

23. Sforza, D.M., Löhner, R., Putman, C., Cebral, J.R.: Hemodynamic analysis of intracranial aneurysms with moving parent arteries: Basilar tip aneurysms. Int J Num Meth Biomed Eng 26 (2010) 1219-1227

24. Thiriet, M.: Biology and Mechanics of Blood Flows, part I: Biology of Blood Flows, part II: Mechanics and Medical Aspects of Blood Flows. Springer (2008)

25. Pironeau, O.: On the transport-diffusion algorithm and its applications to the Navier-Stokes equations. Numer Math 38 (1982) 309-332 
26. Sheng, Z., Thiriet, M., Hecht, F.: A high-order scheme for the incompressible Navier-Stokes equations with open boundary condition. Int J Numer Meth Fl 73 (2013) 58-73

27. Guermond, J.L., Shen, J.: A new class of truly consistent splitting schemes for incompressible flows. J Comput Phys 192 (2003) 262-276

28. Hecht, F.: New development in Freefem++. J Num Math 20 (2012) 251-265

29. Ethier, C.R., Steinman, D.A.: Exact fully 3D Navier-Stokes solutions for benchmarking. Int J Numer Meth Fl 19 (1994) 369-375

30. Gisolf, J., van Lieshout, J.J., van Heusden, K., Pott, F., Stok, W.J., Karemaker, J.M.: Human cerebral venous outflow pathway depends on posture and central venous pressure. J Physiol 560 (2004) 317-327

31. Ford, M.D., Stuhne, G.R., Nikolov, H.N., Habets, D.F., Lownie, S.P., Holdsworth, D.W., Steinman, D.A.: Virtual angiography for visualization and validation of computational models of aneurysm hemodynamics. IEEE T Med Imaging 24 (2005) 1586-1592 